\title{
Algorithm Research and Simulation of Robot Inverse Kinematics Solution
}

\author{
Chengxian $\mathrm{Zhou}^{1}$, Wei $\mathrm{Fu}^{1}$, Ping $\mathrm{Lin}^{1}$ \\ ${ }^{1}$ Departme School of Electric Engineering \& Automation, Xiamen University of Technology, \\ Xiamen 361024, China \\ aemail: zchx258@163.com
}

Keywords: Simulation; Inverse Kinematics; ADAMS

\begin{abstract}
Based on the research prototype of six-joint robot, Googol GRB3016, the virtual prototyping model was built by utilizing the dynamics analytic software ADAMS of mechanical system. This article sets up the constraint parameters and carries out the inverse kinematics simulation of the manipulators' motion along the space spiral trajectory. The curve of location, angular velocity and accelerated velocity are worked out in the post-processing module. The simulation shows manipulators' movement is continuous and stable as well as has no obvious impact. The design accuracy of robots is improved through 3D simulation research which well solved the difficulty of precise parameters in the design of robot body.
\end{abstract}

\section{Introduction}

The robot technology is continuously developing with the wide-scale use of robots for the products processing and maintenance in manufacturing enterprises at home and abroad. Robots with 6-SPS series mechanism, have high flexibility and wide working space, can slick avoid the obstacle, are widely used in the craft process of arc welding and paint spraying [1][2] .With the widely application of robots, it seems especially important to study the basic theory, concrete structure and design technique[3]. The kinematics of robots with six degrees of freedom tandem joints is complicated [4][5]. In order to meet the demand of controller design and dynamic simulation, this article develops the kinematics analysis of robots with 6-SPS series mechanism and gains the forward kinematics by D-H method and plans the motion trail of the end of robots, then makes simulation in ADMS through whose data and curves to learn that the intersection angle, velocity and accelerated velocity consistent with the actual situation so that to affirm the previous structure design and validate the correctness of motion trail and planning, which provides with effective theoretical data and reference basis for the development of the physical prototype.

\section{Establishing the kinematic equation of robots}

Googol GRB3016 type robot is the main study object and establishing the coordinate system of all the joints by D-H method. Through which we get the link parameters illustrated as table 1 .

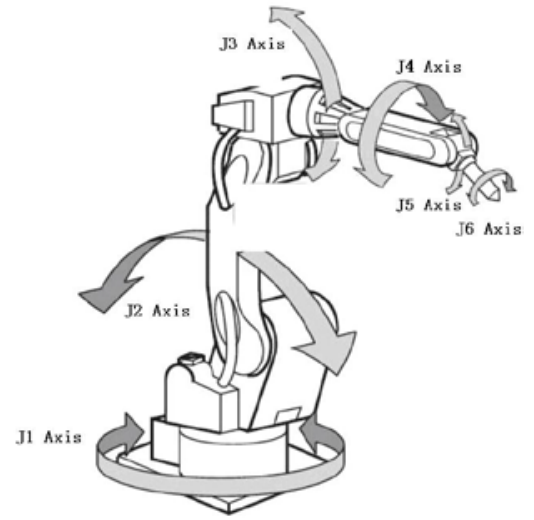

Figure 1 Googol GRB3016 robot 
Table 1 Robot link parameters

\begin{tabular}{|c|c|c|c|c|c|}
\hline Joint i & Variable $\theta_{i}$ & $\alpha_{i-1}$ & $a_{i-1}$ & $d_{i}$ & Variable Scope \\
\hline 1 & $\theta_{1}$ & 0 & 0 & 0 & $-150^{\circ} \sim 150^{\circ}$ \\
\hline 2 & $\theta_{2}$ & $90^{\circ}$ & $a_{1}$ & 0 & $-80^{\circ} \sim 65^{\circ}$ \\
\hline 3 & $\theta_{3}$ & 0 & $a_{2}$ & 0 & $-80^{\circ} \sim 80^{\circ}$ \\
\hline 4 & $\theta_{4}$ & $-90^{\circ}$ & $a_{3} a_{3}$ & $d_{4}$ & $-175^{\circ} \sim 175^{\circ}$ \\
\hline 5 & $\theta_{5}$ & $-90^{\circ}$ & 0 & 0 & $-110^{\circ} \sim 110^{\circ}$ \\
\hline 6 & $\theta_{6}$ & $90^{\circ}$ & 0 & $d_{6}$ & $-200^{\circ} \sim 200^{\circ}$ \\
\hline
\end{tabular}

$a_{1}=150 \mathrm{~mm}, a_{2}=570 \mathrm{~mm}, a_{3}=150 \mathrm{~mm}, d_{4}=650 \mathrm{~mm}, d_{6}=105 \mathrm{~mm}$

The transformation matrix between two links from D-H method:

$$
\begin{aligned}
& { }^{i-1} T_{i}=\left[\begin{array}{cccc}
c \theta_{i} & -s \theta_{i} & 0 & \alpha_{i-1} \\
s \theta_{i} c \alpha_{i-1} & c \theta_{i} c \alpha_{i-1} & -s \alpha_{i-1} & -d_{i} s \alpha_{i-1} \\
s \theta_{i} s \alpha_{i-1} & c \theta_{i} s \alpha_{i-1} & c \alpha_{i-1} & d_{i} c \alpha_{i-1} \\
0 & 0 & 0 & 1
\end{array}\right] \\
& { }^{0} T_{1}=\left[\begin{array}{cccc}
c \theta_{1} & -s \theta_{1} & 0 & 0 \\
s \theta_{1} & c \theta_{1} & 0 & 0 \\
0 & 0 & 1 & 0 \\
0 & 0 & 0 & 1
\end{array}\right] \quad{ }^{1} T_{2}=\left[\begin{array}{cccc}
c \theta_{2} & -s \theta_{2} & 0 & a_{1} \\
0 & 0 & -1 & 0 \\
s \theta_{2} & c \theta_{2} & 0 & 0 \\
0 & 0 & 0 & 1
\end{array}\right] \\
& { }^{2} T_{3}=\left[\begin{array}{cccc}
c \theta_{3} & -s \theta_{3} & 0 & \mathrm{a}_{2} \\
s \theta_{3} & c \theta_{3} & 0 & 0 \\
0 & 0 & 1 & 0 \\
0 & 0 & 0 & 1
\end{array}\right] \quad{ }^{3} T_{4}=\left[\begin{array}{cccc}
c \theta_{4} & -s \theta_{4} & 0 & \mathrm{a}_{3} \\
0 & 0 & 1 & \mathrm{~d}_{4} \\
-s \theta_{4} & -c \theta_{4} & 0 & 0 \\
0 & 0 & 0 & 1
\end{array}\right] \\
& { }^{4} T_{5}=\left[\begin{array}{cccc}
c \theta_{5} & -s \theta_{5} & 0 & 0 \\
0 & 0 & 1 & 0 \\
-s \theta_{5} & -c \theta_{5} & 0 & 0 \\
0 & 0 & 0 & 1
\end{array}\right] \quad{ }^{5} T_{6}=\left[\begin{array}{cccc}
c \theta_{6} & -s \theta_{6} & 0 & 0 \\
0 & 0 & -1 & -d_{6} \\
s \theta_{6} & c \theta_{6} & 0 & 0 \\
0 & 0 & 0 & 1
\end{array}\right]
\end{aligned}
$$

The transformation matrix between two links from D-H method Based on the relation of the connecting rod coordinate department of substrate to the manipulator establish the kinematics equations:

$$
{ }^{0} T_{6}=\left[\begin{array}{cccc}
n & 0 & a & P \\
0 & 0 & 0 & 1
\end{array}\right]={ }^{0} T_{1}^{1} T_{2}{ }^{2} T_{3}{ }^{3} T_{4}{ }^{4} T_{5}{ }^{5} T_{6}=\left[\begin{array}{cccc}
n_{x} & o_{x} & a_{x} & P_{x} \\
n_{y} & o_{y} & a_{y} & P_{y} \\
n_{z} & o_{z} & a_{z} & P_{z} \\
0 & 0 & 0 & 1
\end{array}\right]
$$

There exist a lot of solution methods of robot inverse kinematics at home and abroad such as numerical method, geometrical method and analytic method, iteration method, numerical simulation. Under the limitation of joint variable motion range situation, the analytic solution can get the unique solution. The complexity degree inverse kinematics has much to do with the robot structure. In most cases, the most industrial robots are designed with the simple dynamical model to present the inverse kinematics with closed form. The Googol GRB3016 has the closed solution for it meet the 
Pieper norm. Based on the normal solution, the idea of deducing the formula of inverse kinematics is multiplied by transformation matrix inverse matrix in the both sides of equation. An unknown number is moved from the right of matrix to the left, isolating the variable and working out the unknown number. And then moving the next unknown number to the left and repeat the last process until figure out all the unknown number. The detailed derivation process will not be listed here. It may has multi-group algebraic solution. However, according to the space scope of operation and mechanical structure, we can exclude the interference solutions to get the unique solution.

This article solves the formula of the robot inverse kinematics with ADAMS software and spot motor function. Firstly, creating a general spot drive with 6 freedom degree in the robot end effector. And then we respectively to define a functional equation with time as the free variable. The general spot drive leads the axis to move. Afterwards setting the type, time and step width of simulation to carry on the simulation with each other as well as gain the movement curve of all joints by the measure function of ADAMS software. After that we turn the movement curve into spline curve in the processing module after ADAMS. Finally, adding spline driving function on every axle so that to get the robot end-effector's simulation curve of movement locus. At last the positive and inverse solution process of robot kinematics is finished. The solution process of robot kinematics is shown in figure 2.

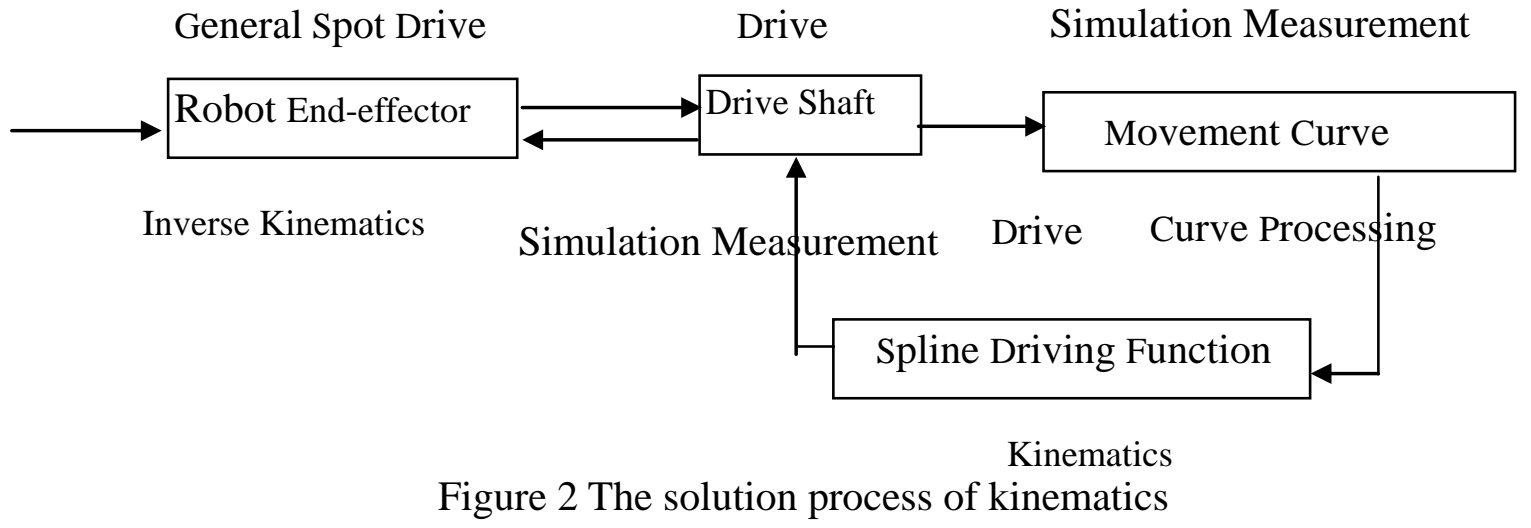

In accordance with the sequence from foundation to the end of actuator, every mechanical arm is a part. In the line with the principle of simples as much as possible on the basis of guarantee the simulated effect, we are supposed to impose various constraints on components and check every so often to see whether the constraints are right. We are expected to pay attention to the selecting sequence when creating the constraints. The two constraint parts in ADAMS, the part 1 should connect to the part 2 and the relative movement restrained is part 1 to part 2 .

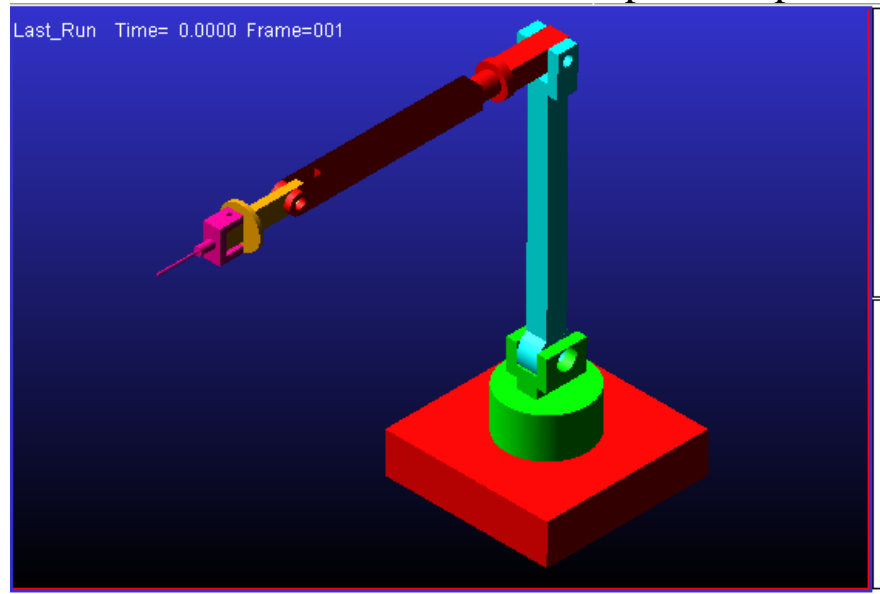

Figure 3. Three-dimensional isometry diagram of simulation model

Choosing the Model Verify in Tools Menu in the establishment model and testing the model freedom. Clicking on the General Motion icon in drivers library. And then carrying out the self-inspection after modeling so that to verify the validity the validity of virtual 


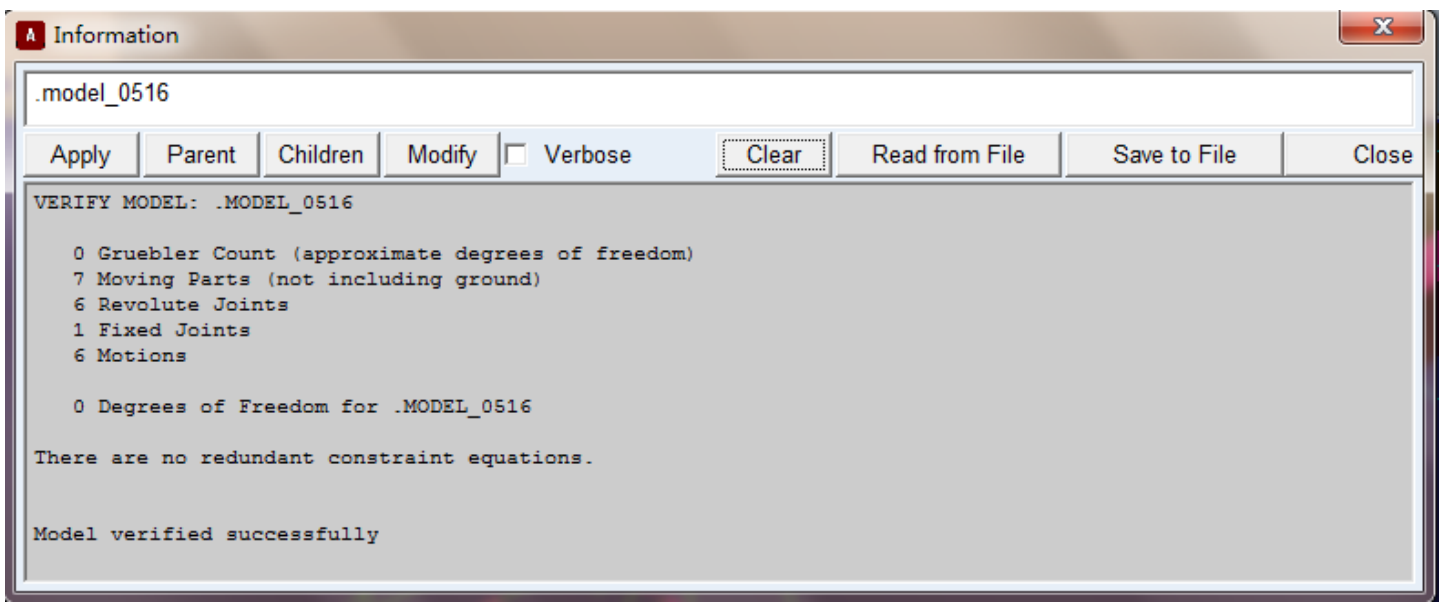

Figure 4. Model self-check result

From the examination report, we learn that the prototype has one fixed joints, six revolution joints, six drivers, seven assembly parts and has no degrees of freedom. There is not redundancy constraints and the checking result is correct as well as meet the design requirements. During the progress of simulation the ADAMS/View works out the kinetic characteristics automatically such as the curves of distance, speed, acceleration, torque and energy etc.. Choosing the information needed to observe the change of curves.

\section{Simulation of Robot Kinematics}

Adding a general point driver at the end like the figure 5. The driver function equation is illustrated in the figure 6 .

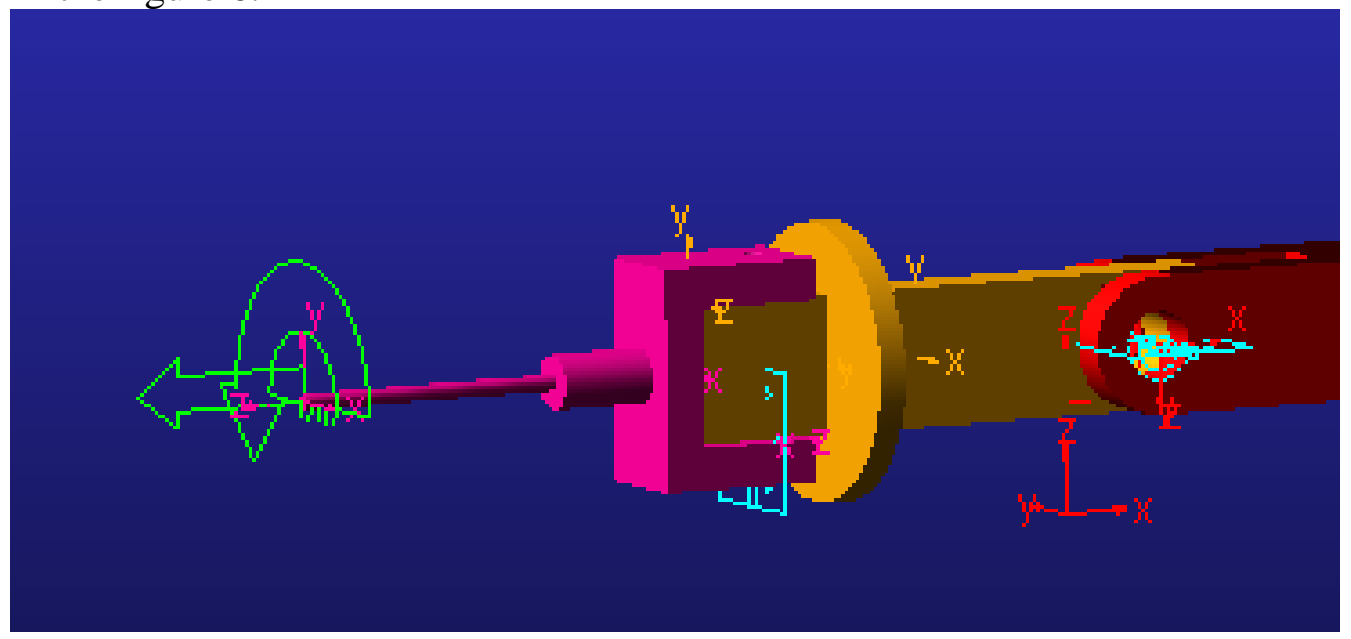

Figure 5 Sketch map of point driver

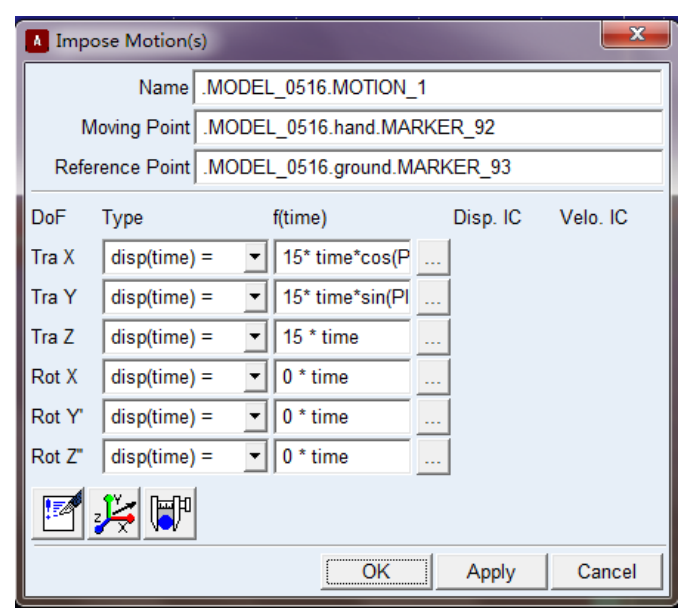

Figure 6 Driver function equation 
Choosing the option of simulation enter into simulation.The simulation options in tool bar are listed in figure6. Clicking on the icon or pressing F8 into the post-processing module.

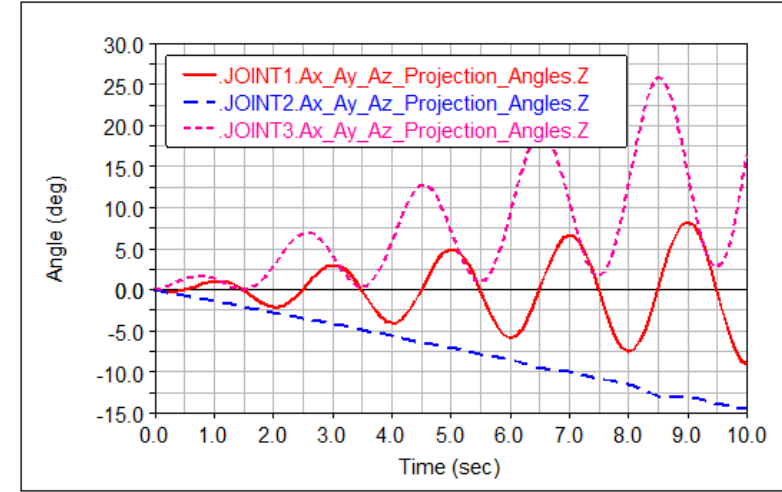

Figure 7 Curve of rotating angle for joint 1-3

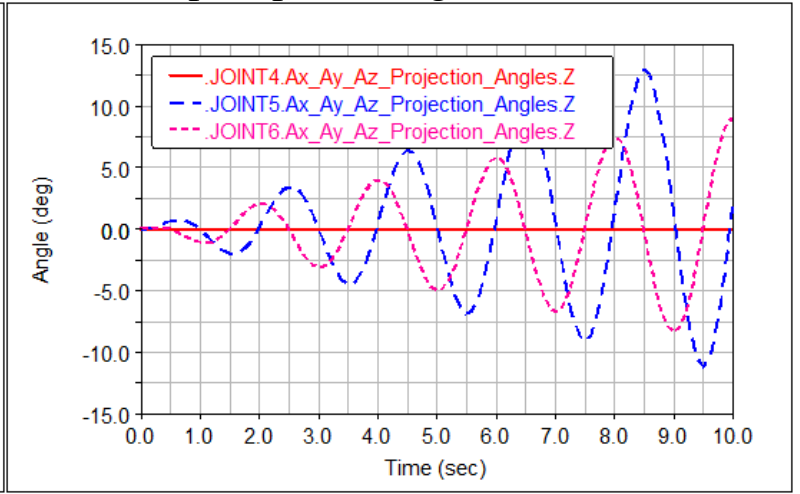

Figure 8 Curve of rotating angle for joint 4-6

Adding the spline driving function for every joint,collecting the data sample points, generating spline function firstly delete point drivers and then add the spline driver in the later constrains. Clicking on the right mouse button to choose Modify option, which shown in the figure 9

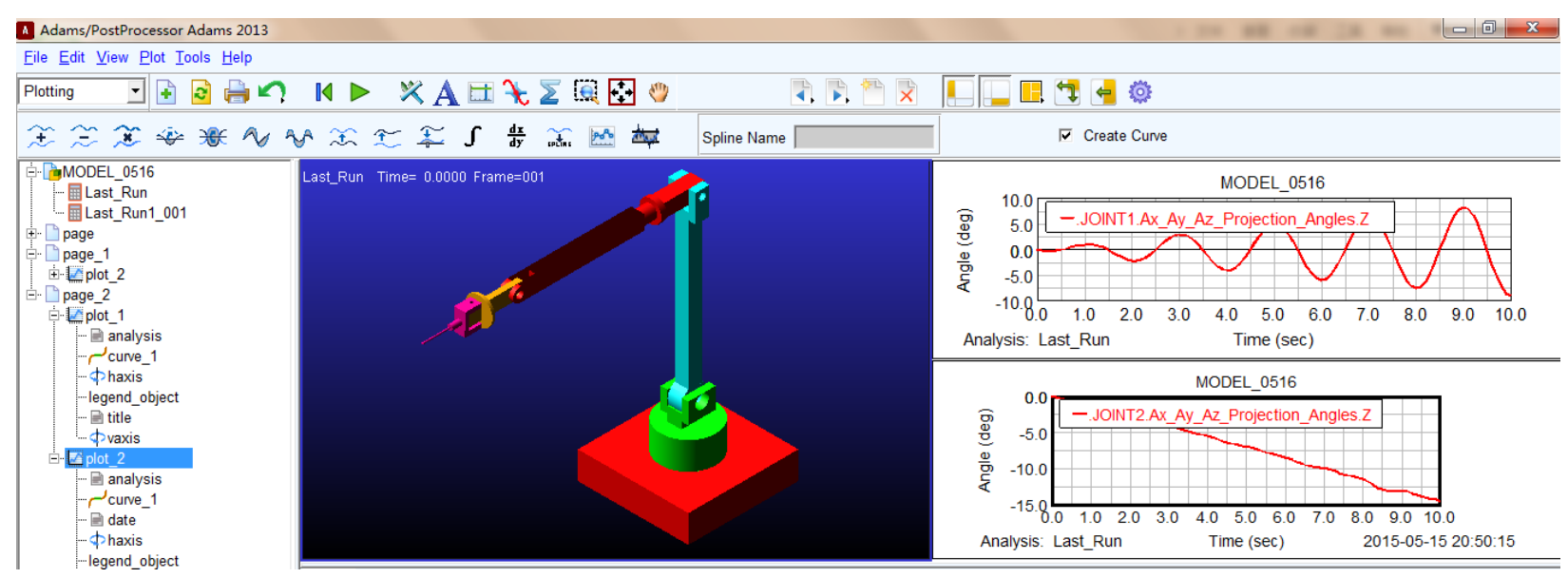

Figure 9 Cresting the spline curve

\section{The analysis of simulation result}

Comparing the manipulator's end effector of inverse solution with that of normal solution through which we learn that applying ADAMS to solve inverse kinematics is simpler and more intuitional than theoretical derivation. Besides, the error is close to zero, which shows that utilizing ADAMS for solving normal and inverse kinematics is feasible and accurate.

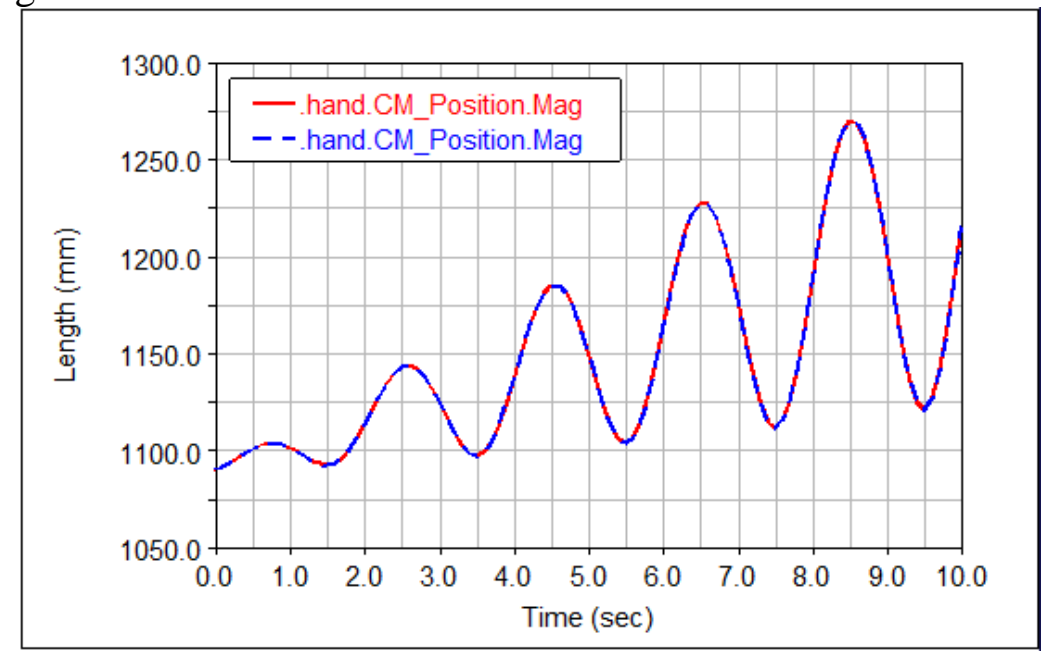

Figure 10 Error analysis 


\section{Conclusion}

Robot kinematics is complex and worth researching problem. In order to control the location and gesture of robots, carrying out the interactive simulation verification of kinematics for robots is necessary. Adopting the $\mathrm{D}-\mathrm{H}$ method to get the normal solution of kinematics, taking advantage the ADAMS/View to create virtual prototyping model of Googol GRB3016 robots so that to establish the prototype model of actual machine.Through setting related simulation parameters to make simulation. The track of spline planning of robot is smooth and fluent by observe the position and speed of kinematics, angular velocity parameters and simulation curves. The simulation result prove that the method of establishing six degrees of freedom control system simulation model through ADAMS software is practicable. Moreover, according to the parameters we can make use of the optimization function of ADAMS system to choose the prioritization scheme.We can use optimization function to establish a more perfect model in the later researches. Therefore, the 3D simulation research has important significance for the development of robot body and improvement of robot performance quickly and accurately.

\section{Acknowledgement}

In this paper, the research was sponsored by the Nature Science Foundation of Fujian Province (Project No. 2014J01254) and Project of Education Department of Fujian Province (Project No. JA14232)

\section{References}

[1]J.Norberto Pires,A. Loureiro,T.Godinho,P.Ferreira,B.Fernando,and J. Morgado, "Welding robots," Robotics \&Automation Magazine, IEEE, vol. 10, pp. 45-55, 2003.

[2]QUAN Jian-hui, DENG Yu-wen. Analysis of Peak Torque of SCARA Robot Based on ADAMS[J]. Journal of Luoyang Institute of Science and Technology,2013,23(4):29-33.

[3]Wang K,Lien T K.The structure design and kinematics of a robot manipulator[J].robotics and Computer-Integrated Manufacturing,2005,1(5):84-89.

[4]H.J. Kang,J.Y. Park.Work planning using genetic algorithm and 3Dsimulation at a subassembly line of shipyard[R]. IEEE 2004:218-222.

[5]W.Schiehlen, Recent developments in multibody dynamics, Joural of Mechanical Science and Technology 19 (1) (2005) 227-236.

[6] Ruan Peng,Yu Zhiwei,Zhang Hao,etal Gait Planning and Simulation of Gecko Inspired Robot Based on ADAMS[J].Robot,2010,32(4):499-509. 